\title{
Maintenance pharmacotherapy for recurrent major depressive disorder in primary care : A 5-year follow-up study
}

\section{Riihimäki, K.}

2017-03

Riihimäki , K, Vuorilehto , M \& Isometsa , E 2017 , ' Maintenance pharmacotherapy for recurrent major depressive disorder in primary care : A 5-year follow-up study ' , European Psychiatry, vol. 41, pp. 111-114 . https://doi.org/10.1016/j.eurpsy.2016.10.010

http://hdl.handle.net/10138/234696

https://doi.org/10.1016/j.eurpsy.2016.10.010

publishedVersion

Downloaded from Helda, University of Helsinki institutional repository.

This is an electronic reprint of the original article.

This reprint may differ from the original in pagination and typographic detail.

Please cite the original version. 
Original article

\title{
Maintenance pharmacotherapy for recurrent major depressive disorder in primary care: A 5-year follow-up study
}

\author{
K. Riihimäki ${ }^{\text {a,c }}$, M. Vuorilehto ${ }^{\mathrm{a}, \mathrm{b}}$, E. Isometsä ${ }^{\mathrm{a}, \mathrm{b}, *}$ \\ a Mental Health Unit, National Institute for Health and Welfare, Helsinki, Finland \\ ${ }^{\mathrm{b}}$ Department of Psychiatry, University of Helsinki, Helsinki University Hospital, Helsinki, Finland \\ ${ }^{\mathrm{c}}$ Health Care and Social Services, City of Järvenpää, Järvenpää, Finland
}

\section{A R T I C L E I N F O}

\section{Article history:}

Received 16 September 2016

Received in revised form 27 October 2016

Accepted 28 October 2016

Available online 27 January 2017

Keywords:

Depression

Maintenance pharmacotherapy

Primary care

\begin{abstract}
A B S T R A C T
Background: Most practice guidelines recommend maintenance antidepressant treatment for recurrent major depressive disorder. However, the degree to which such guidance is actually followed in primary health care has remained obscure. We investigated the provision of maintenance antidepressant treatment within a representative primary care five-year cohort study.

Methods: In the Vantaa Primary Care Depression Study, a stratified random sample of 1119 adult patients was screened for depression using the Prime-MD. Depressive and comorbid psychiatric disorders were diagnosed using SCID-I/P and SCID-II interviews. Of the 137 patients with depressive disorders, $82 \%$ completed the prospective five-year follow-up. A graphic life chart enabling evaluation of the longitudinal course of episodes plus duration of pharmacotherapies was used. In accordance with national guidelines, an indication for maintenance treatment was defined to exist after three or more lifetime major depressive episodes (MDEs); maintenance treatment was to commence four months after onset of full remission.

Results: Of the cohort patients, 34\% (46/137) had three or more lifetime MDEs, thus indicating the requirement for maintenance pharmacotherapy. Of these, half $(54 \%, 25 / 46)$ received maintenance treatment, for only $29 \%$ (489/1670) of the months indicated.

Conclusions: In this cohort of depressed primary care patients, half of patients with indications for maintenance treatment actually received it, and only for a fraction of the time indicated. Antidepressant maintenance treatment for the prevention of recurrences is unlikely to be subject to large-scale actualization as recommended, which may significantly undermine the potential public health benefits of treatment.
\end{abstract}

(c) 2016 Elsevier Masson SAS. All rights reserved.

\section{Introduction}

Depressive disorders are mostly evaluated and treated in primary care settings, where chronic and recurrent depressions are common [1]. Most practice guidelines produced by national health care organizations or professional societies recommend antidepressant maintenance pharmacotherapy for patients with three or more major depressive episodes (MDEs) [2-5]. However, the degree to which such guidance is actually followed in primary care has remained obscure. Although registry-based studies [6,7] indicate a large proportion of patients receiving long-term

\footnotetext{
* Corresponding author. Department of Psychiatry, Faculty of Medicine, University of Helsinki, P.O. Box 22, 00014 Helsinki, Finland.

Tel.: +3589471 63728; fax: +358947163735.

E-mail address: erkki.isometsa@hus.fi (E. Isometsä).
}

antidepressant medication, indications for patients' long-term treatment remain unclear. Long-term antidepressant treatment may reflect the chronicity of depression, nonspecific long-term use of antidepressants, deliberate maintenance treatment, or antidepressant treatment for other indications. A major methodological obstacle for clinical-epidemiological studies in this field is that evaluating continuity and adequacy of maintenance treatment of depression necessitates both determining treatment phases (acute, continuation or maintenance) using labor-intensive life-chart methodology, and concurrently incorporating accurate data on the temporal course of pharmacological treatment. Therefore, such studies are scarce. In our previous life-chart-based study from secondary care regional psychiatric services, we documented major shortcomings in the implementation of maintenance treatment [8].

To our knowledge, no primary care study has examined how recommendations for antidepressant maintenance treatment are 
implemented. We investigated its prevalence, duration and predictors, and roles of attitudes and adherence in a naturalistic prospective long-term Finnish cohort of primary care patients with depressive disorders.

\section{Material and methods}

\subsection{Patients and procedures}

The methodology of the Vantaa Primary Care Depression Study (PC-VDS) has been published in detail elsewhere [9,10]. In brief, based on stratified sampling, altogether 373 of 1119 general practitioners' (GP) patients aged 20-69 screened with the PRIMEMD had a positive screen for depression. The presence of at least one core symptom of major depressive disorder (MDD) according to the Structured Clinical Interview for DSM-IV Axis I Disorders (SCID-I/P) [11] was confirmed by telephone. All of the 175 potentially eligible patients were interviewed face-to-face using the SCID-I/P with psychotic screen. Inclusion criteria were current (1) MDD, (2) dysthymia, (3) subsyndromal MDD with two to four depressive symptoms (minimum one core symptom) and lifetime MDD and (4) minor depression otherwise similar to subsyndromal MDD, but without an MDD history. Refused (15\%) and consented patients did not differ significantly in age or gender. The diagnostic reliability for current depressive disorder was excellent (kap$\mathrm{pa}=1.0$ ). The PC-VDS was approved by the pertinent Ethics Committee in 2001.

The final sample comprised 137 patients. Current and lifetime psychiatric disorders were assessed with SCID-I/P and SCID-II interviews $[11,12]$. In addition to face-to-face interviews, observer- and self-report scales and all medical and psychiatric records were used to assess retrospective and prospective course of depression, comorbid disorders and psychosocial and socioeconomic factors. After baseline, patients were prospectively investigated at 3, 6 and 18 months and 5 years [10]. The 5-year investigation included all the same diagnostic interviews, scales and records as at baseline. The timing and duration of episodes of depression (MDEs and partial and full remission) and indications for acute, continuation and maintenance antidepressant treatment were integrated into a graphic life chart. We defined maintenance indication to exist after three or more lifetime MDEs (before or during the follow-up) and then achieved full remission for more than two months; treatment was to commence four months after the onset of full remission (National Finnish Current Care Guideline 2016). We have previously reported methodology and findings related to acute phase treatment [13].

Of the 137 patients initially included in the study, 127 (93\%) participated in the 18-month and 112 (82\%) in the 5-year followup. Patients remained in the cohort until they were censored due to change of diagnosis to bipolar disorder $(4 \%, 5 / 137)$ or death $(4 \%, 6$ / 137). The final follow-up group consisted of the 134 patients with some follow-up information, 110 of them had had lifetime MDD. The dropouts (18\%) did not significantly differ from participants in terms of socio-demographic or clinical features [10].

\subsection{Measures of attitudes, adherence and contacts with general practitioners (GP)}

Attitudes towards different treatments were rated very positive, positive, neutral, negative and very negative, and analyzed in two groups: favorable and negative. Patient adherence to antidepressants was rated (1) regularly, (2) somewhat irregularly (whether this would affect treatment goals was unknown), (3) very irregularly (treatment did not proceed according to plan), or (4) not at all (provided treatment could not be implemented). All contacts with GPs concerning any health problems were totaled; the monitoring comprised all contacts where depression-related symptoms or treatments were discussed. The primary reasons for poor adherence were classified into patient-, GP- and organizationrelated factors with a semi-structured questionnaire, based on interviews and records.

\subsection{Statistical methods}

Between-group comparisons were carried out using Fisher's exact test, the two-sample $t$-test, and the Mann-Whitney or Kruskal-Wallis tests. Logistic and linear regression models were used to investigate associations of different variables; models were controlled for age, gender and duration of maintenance indication, and in the final models, the non-significant variables were omitted. SPSS, version 23, was used.

\section{Results}

\subsection{Proportion and duration of indicated and received maintenance antidepressant treatment during the 5-year follow-up}

Altogether $34 \%(46 / 137)$ of the cohort patients had three or more lifetime MDEs, and thus an indication for maintenance pharmacotherapy. Half $(54 \%, 25 / 46)$ of them actually received it. The most frequently prescribed antidepressants were selective serotonin reuptake inhibitors. Characteristics of patients with an indication of maintenance, comparing those who received it with those who had not, are presented in Table 1.

Among the 46 patients, maintenance treatment would have been indicated for a mean 36.3 months (median 41.8, SD 20.6, 174 months). However, the mean observed duration of maintenance treatment was 19.6 months (median 12.0, SD 18.0, 1-60 months). It covered 29\% (489/1670 months) of the time the indication was in force (Fig. 1).

\subsection{Follow-up contacts with general practitioners (GP) and psychosocial treatments during the 5-year follow-up}

During the follow-up, the patients visited GPs overall (for any reason) for a mean of 19 times (median $13, S D \pm 21,0-153$ ). The mean number of GP contacts due to depression was 8 (median 4 , $\mathrm{SD} \pm 12,0-83$ ). A fourth (24\%) of the patients also sought psychiatric care, often ambulatory. One fifth (20\%) were treated in psychiatric outpatient units and one tenth $(11 \%)$ received inpatient care. Some kind of psychosocial support other than that from a GP was offered to one third (38\%); one fifth (20\%) actually received it.

\subsection{Patients' attitudes and adherence to antidepressant treatment}

Patients' attitudes to antidepressants were very positive in $29 \%$, positive in $42 \%$, neutral in $17 \%$, negative in $7 \%$ and very negative in $5 \%$; to maintenance in $26 \%, 36 \%, 23 \%, 8 \%$ and $8 \%$, respectively, and for comparison, to psychotherapeutic support in $45 \%, 40 \%, 13 \%, 3 \%$ and $0 \%$. There were no statistically differences in attitudes towards different treatments.

The patients receiving maintenance antidepressant treatment had reportedly adhered to it regularly in $29 \%$, moderately in $25 \%$, incompletely in $23 \%$ and not at all in $23 \%$ of the cases.

The reasons for poor adherence included patient-related factors in $37 \%$, GP-related factors in $43 \%$ and organization-related factors in $20 \%$ of the cases. Patients-related factors included inability to adhere, lack of motivation, negative attitudes, recovery, and inadequate effects, side effects or costs of antidepressants. GPrelated factors included incomplete follow-up (41\%), antidepressant not offered (20\%), inadequately small dosage (0\%), dosage not increased (10\%), antidepressant not changed (3\%), maintenance 
Table 1

Characteristics of depressive patients with indicated maintenance antidepressant (AD) treatment in the Vantaa Primary Care Depression Study ( $n=46 / 137$ ).

\begin{tabular}{|c|c|c|c|c|c|c|c|}
\hline & \multicolumn{2}{|c|}{$\begin{array}{l}\text { Maintenance AD, } \\
n=25\end{array}$} & \multicolumn{2}{|c|}{$\begin{array}{l}\text { No maintenance, } \\
n=21\end{array}$} & \multicolumn{2}{|l|}{ Total } & \multirow[t]{2}{*}{$P$} \\
\hline & $n$ & $\%$ & $n$ & $\%$ & $n$ & $\%$ & \\
\hline \multicolumn{8}{|l|}{ Socio-demographic features } \\
\hline Female & 21 & 84 & 19 & 91 & 40 & 63 & 0.521 \\
\hline Married or cohabiting & 8 & 32 & 12 & 57 & 20 & 44 & 0.090 \\
\hline Vocational education, any & 17 & 68 & 16 & 76 & 33 & 72 & 0.543 \\
\hline Employed & 15 & 60 & 7 & 33 & 22 & 48 & 0.067 \\
\hline Welfare benefit received & 10 & 40 & 3 & 14 & 13 & 28 & 0.055 \\
\hline \multicolumn{8}{|l|}{ Clinical features } \\
\hline Comorbid psychiatric disorder (any) & 21 & 84 & 10 & 48 & 31 & 67 & 0.008 \\
\hline Axis I, any & 19 & 76 & 7 & 33 & 26 & 57 & 0.005 \\
\hline Anxiety disorder, any & 14 & 44 & 5 & 24 & 19 & 41 & 0.029 \\
\hline Cluster $\mathrm{C}$ personality disorder & 12 & 48 & 4 & 19 & 16 & 35 & 0.042 \\
\hline Borderline personality disorder & 6 & 24 & 5 & 24 & 11 & 24 & 0.988 \\
\hline Substance use disorder & 2 & 8 & 2 & 10 & 4 & 9 & 0.857 \\
\hline \multicolumn{8}{|l|}{ Treatment-related factors } \\
\hline Positive attitude to maintenance $\mathrm{AD}$ & 10 & 63 & 10 & 83 & 20 & 71 & 0.236 \\
\hline Good adherence to ADs & 7 & 35 & 0 & 0 & 7 & 28 & 0.127 \\
\hline Organization-related reasons to shorter maintenance & 9 & 56 & 4 & 80 & 13 & 62 & 0.365 \\
\hline Psychosocial treatment during the 5-year follow-up & 17 & 71 & 2 & 10 & 19 & 46 & $<0.001$ \\
\hline \multirow[t]{2}{*}{ Psychiatric care during follow-up } & 7 & 28 & 2 & 10 & 9 & 20 & 0.120 \\
\hline & Mean & SD & Mean & SD & Mean & SD & $P$ \\
\hline \multicolumn{8}{|l|}{ Socio-demographic features } \\
\hline Age (years) & 42.8 & 14.4 & 42.2 & 13.8 & 42.5 & 13.8 & 0.886 \\
\hline \multicolumn{8}{|l|}{ Clinical features } \\
\hline Beck depression inventory & 18.9 & 8.7 & 14.5 & 10.2 & 16.9 & 9.6 & 0.121 \\
\hline Beck anxiety inventory & 15.6 & 11.0 & 13.1 & 12.5 & 14.5 & 11.6 & 0.121 \\
\hline Social and Occupational Functioning Assessment Scale & 60.6 & 10.5 & 57.6 & 10.3 & 59.2 & 10.4 & 0.340 \\
\hline Neuroticism (EPI-Q) & 7.0 & 1.8 & 5.6 & 2.5 & 6.3 & 2.2 & 0.030 \\
\hline Number of MDEs & 6.0 & 4.3 & 3.5 & 4.2 & 4.9 & 4.4 & 0.002 \\
\hline Time spent in MDE during 5 years (months) & 15.8 & 14.5 & 6.5 & 10.1 & 11.5 & 13.4 & 0.009 \\
\hline Time spent in full remission (months) & 35.3 & 18.7 & 46.6 & 18.9 & 40.5 & 19.4 & 0.050 \\
\hline \multicolumn{8}{|l|}{ Treatment-related factors } \\
\hline Maintenance indication duration during 5 years (month) & 32.5 & 19.0 & 41.0 & 22.3 & 36.4 & 20.6 & 0.123 \\
\hline GP contacts due to depression & 10.6 & 9.5 & 7.1 & 15.0 & 9 & 12.3 & 0.005 \\
\hline
\end{tabular}

Fisher's exact test, the two-sample t-test, and the Mann-Whitney or Kruskal-Wallis tests.

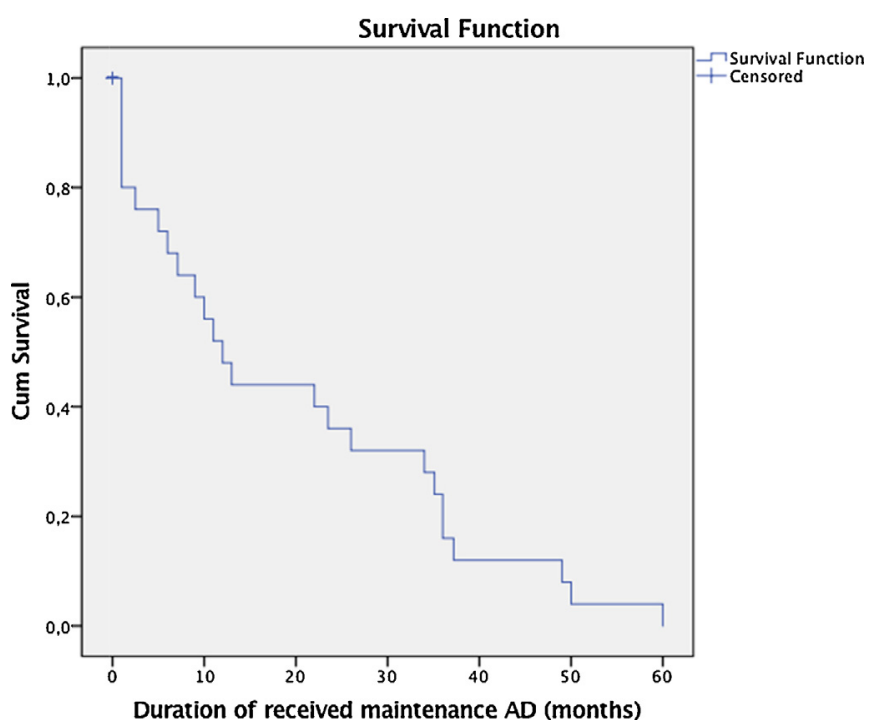

Fig. 1. The duration of indicated and received maintenance antidepressant treatment estimated by survival curve during the 5-year follow-up in the Vantaa Primary Care Depression Study. shorter than 4 months (9\%), not remitted to psychiatric care (1\%), undetected substance abuse (4\%), and inadequate communication (11\%). Organization-related factors included difficulties to get an appointment, long waiting times for psychiatric care and changes in attending personnel.

\subsection{Predictors for maintenance treatment}

In univariate logistic regression analyses, from baseline variables (adjusted for age and gender), anxiety disorders, cluster $C$ personality disorder and neuroticism associated with received maintenance. However, in multivariate logistic regression, only anxiety disorders associated with prescribed maintenance treatment (OR 0.251, B $-1.384, P=0.037,95 \%$ CI $0.068-0.921$ ). In univariate linear regression analyses, the results were similar. In multivariate linear regression, from baseline variables (adjusted for age, gender and duration of maintenance indication), duration of received maintenance associated significantly with anxiety disorders (B 13.571, $P=0.013,95 \% \mathrm{CI} 3.064-24.077$ ). Of the followup variables, having received psychosocial treatment associated with more and longer maintenance pharmacotherapy.

\section{Discussion}

We investigated the provision of antidepressant pharmacotherapy for recurrent depression in a screening-based, representative 
regional cohort of primary care (PC) patients with depressive disorders. During the five-year follow-up, half of our patients with three or more MDEs actually received maintenance antidepressant treatment, for only a fraction of the time indicated. Despite generally positive attitudes, only a third of even those receiving maintenance antidepressants reported good adherence to it.

The major strength of the study was the use of a life chart, which enabled to evaluate not only the time spent in different states of depression (MDE and partial and full remission) but also the durations of different treatment indications (acute, continuation and maintenance) and all treatments received [10]. Further strengths included the screening-based cohort from a stratified sampling of 1119 patients, structured interviews with SCID-I/P and SCID-II by psychiatrists and the longitudinal study design with a five-year follow-up, and a small dropout rate. All primary and psychiatric care records were available. Patient attitudes towards treatment, adherence and attributed reasons for poor adherences were investigated. The naturalistic study design reflects the actual patient load of the local GPs.

Nevertheless, some limitations need to be noted. First, despite extensive screening of a large number of patients [9], the size of the cohort ( $n=137$ ) remained moderate, and number of patients with an indication for maintenance antidepressant treatment $(n=46)$ still smaller. This renders the study somewhat vulnerable to type II errors and risk of spurious findings. Second, because our study was performed between 2002-2007, generalisability of findings to current settings remains uncertain. Preceding and at the time of prospective cohort data collection, the GPs were educated about depression. This included use and indications of antidepressant maintenance treatment, recommended by the first national Finnish Current Guideline for depression published in 2004. Third, primary care records may be quite concise, e.g. offered treatment may not have been recorded in cases a patient refused, which could lead to underestimates of treatments offered. Fourth, the attitudes of the attending GPs were not evaluated. Fifth, our analyses focus on whether or not the patients were prescribed antidepressants during a period during which this, based on the definition, was indicated as maintenance treatment. The actual treatment decision may or may not have been based on such considerations. Finally, generalisability of our findings needs to be confirmed by replicating them in other representative studies in other settings.

To our knowledge, no other primary care study has reported results for provision and continuity of maintenance antidepressant pharmacotherapy. In an analysis of the Dutch longitudinal epidemiological NESDA study data, Piek et al. [14] approximated indications and provision of maintenance antidepressant among primary care attenders, finding characteristics of those receiving long-term antidepressant treatment not fully concordant with national guidelines. Our findings replicate those from an earlier similar life-chart-based study [8] conducted in the same area in psychiatric settings. Knowledge of predictors might be helpful for improving care, but when analyzing them, it is difficult to differentiate causes from consequences. Due to confounding by indication, observed predictors may in fact predict only chronic course of depression. Finding longer times of maintenance associated with more psychosocial support is potentially of interest. However, psychosocial support could contribute to antidepressant-adherence or vice versa; achieving full remission and thus an indication for maintenance antidepressants, or patients suffering from chronic or recurrent depression or having positive attitudes to treatment may receive more kinds of treatments.
While not systematically evaluated, our strong impression from the patient interviews and records was, that GPs rarely conceptualized or discussed continuing antidepressant treatment in terms of preventing depressive recurrences, or phases of treatment. Primary care patients contact their GPs for many different, often multiple somatic and psychiatric reasons. Antidepressants were often prescribed along while treating other issues, and patient records may thus have remained incomplete. There were few, if any, deliberate recorded treatment decisions of maintenance treatment.

\section{Conclusions}

In this representative Finnish cohort of depressed primary care patients, we found that half of the patients with indications for maintenance treatment actually received it, and only for a fraction of the time indicated. We expect that antidepressant maintenance treatment for the prevention of recurrences is unlikely to actualize on as large a scale as recommended, which may significantly undermine the potential public health benefits of treatment.

\section{Disclosure of interest}

The authors declare that they have no competing interest.

\section{Acknowledgements}

The City of Vantaa, Academy of Finland, Helsinki University Hospital and The Finnish Psychiatric Association have supported this research by grants.

\section{References}

[1] Gilchrist G, Gunn J. Observational studies of depression in primary care: what do we know? BMC Fam Pract 2007:8:28.

[2] American Psychiatric Association (APA). Practice guideline for the treatment of patients with major depressive disorder; 2010 [psychiatryonline.org].

[3] NICE, editor. Depression: the NICE guideline on the treatment and management of depression in adults (updated edition). London: National Institute for Health and Clinical Excellence; 2010.

[4] The Finnish Medical Society Duodecim, Finnish Psychiatric Association. Current care guideline (Käypä hoito -suositus): Depressio. Suomalaisen Lääkäriseuran Duodecimin ja Suomen Psykiatriyhdistys ry:n asettama työryhmä; 2014.

[5] Kennedy SH, Lam RW, Parikh SV, Patten SB, Ravindran AV, Canadian network for mood and anxiety treatments (CANMAT). Clinical guidelines for the management of major depressive disorder in adults. J Affect Disord 2009;117:1-64.

[6] Sihvo S, Isometsä E, Kiviruusu O, Hämäläinen J, Suvisaari J, Perälä J, et al Antidepressant utilisation patterns and determinants of short-term and nonpsychiatric use in the Finnish general adult population. J Affect Disord 2008:110:94-105.

[7] Coupland C, Hill T, Morriss R, Arthur A, Moore M, Hippisley-Cox J. Antidepressant use and risk of suicide and attempted suicide or self harm in people aged 20 to 64: cohort study using a primary care database. BMJ 2015;350:517.

[8] Holma I, Holma K, Melartin T, Isometsa E. Maintenance pharmacotherapy for recurrent major depressive disorder: 5-year follow-up study. Br J Psychiatry 2008; 193:163-4.

[9] Vuorilehto M, Melartin T, Isometsa E. Depressive disorders in primary care: recurrent, chronic, and co-morbid. Psychol Med 2005;35:673-82.

[10] Riihimaki KA, Vuorilehto MS, Melartin TK, Isometsa ET. Five-year outcome of major depressive disorder in primary health care. Psychol Med 2011:16:1-11.

[11] First MB, Spitzer RL, Gibbon M, Williams JBW, editors. Structured clinical interview for DSM-IV-TR axis I disorders, research version, patient edition with psychotic screen. New York: New York State Psychiatric Institute; 2002

[12] First MB, Gibbon M, Spitzer RL, Williams JBW, Benjamin LS, editors. Structured clinical interview for DSM-IV axis II personality disorders, (SCID-II). Washington, D.C: American Psychiatric Press, Inc; 1997.

[13] Vuorilehto M, Riihimäki K, Isometsä E. Pharmacological and psychosocial treatment of depression in primary care: low intensity and poor adherence and continuity. J Affect Disord 2016;202:145-52.

[14] Piek E, Kollen BJ, van der Meer K, Penninx BW, Nolen WA. Maintenance use of antidepressants in Dutch general practice: non-guideline concordant. PLoS One 2014;23:e97463. 